\title{
The Promise of Single Cell Omics for Onco-therapy
}

Ebrahim Azizi, Shawn G Clouthier and Max S Wicha*

Comprehensive Cancer Center, Department of Internal Medicine, University of Michigan, Ann Arbor, MI, 48109, USA

*Corresponding author: Max S Wicha, Comprehensive Cancer Center, Department of Internal Medicine, University of Michigan, Ann Arbor, MI, 48109, USA, Tel: (734) 615-7434 Fax: (734) 764-1288; E-mail: clouthie@umich.edu

Rec date: Jun 09, 2014, Acc date: Aug 25, 2014, Pub date: Oct 20, 2014

Copyright: $\odot 2014$ Azizi E, et al., This is an open-access article distributed under the terms of the Creative Commons Attribution License, which permits unrestricted use, distribution, and reproduction in any medium, provided the original author and source are credited.

\begin{abstract}
Despite extensive research effort and considerable progress, the "war on cancer" that president Nixon declared in 1971 has yet to be optimally integrated into cancer therapeutics and as such cancer remains a major medical challenge for oncologists. The dynamic and complex biology of tumor cells undergoing clonal evolution generates cells with diverse degrees of drug resistance and metastatic potential. This highlights the need to be able to access this clonal density in order to develop effective therapeutics. With this prospective, early phase single cell studies are vital for thoroughly interrogating tumor heterogeneity to uncover more about cancer cell biology and to explore new therapeutic targets leading to more successful treatments. Current evidence supports the notion that clonogenic cells within the tumor mass may potentially give rise to a population of cells with unique genomic, transcriptomic and proteomic features distinct from the rest of the tumor mass. This observation can explain drug resistance after an initial period of primary tumor response. Therefore, completely abrogating or at a minimum achieving long-term, durable control over cancer requires researchers and oncologists to employ a personalized medicine approach that includes both tumor and patient-associated variables to modify current therapeutic regimens. In this review we discuss the importance of omics and in particular single cell genomics which are increasingly promising given recently developed technology advancements to facilitate exploration of cellular heterogeneity and tumor complexity.
\end{abstract}

Keywords: Cancer genomics; Single cell; Tumor heterogeneity; Oncology therapy

\section{The Combinatorial Nature of Cancer}

Cancer is a catch all term for a collection of many related, but discrete diseases that share some degree of commonality, but can no longer be considered a single disease [1]. Although tumors may initiate following malignant transformation of even a single normal cell, more probably continuous genetic mutations and gene expression alterations during tumor progression lead to a metastatic phenotype composed of multiple subsets of tumor cells with distinct characteristics. In fact, each of the genetic and epigenetic events that happen to the tumor cells after tumor initiation result in a disease that collectively is termed cancer. Yet each discrete cancer shares certain characteristics with other malignancies. In this context, malignancy of tumor cells increase as a result of the cumulative formation of a series of tumor clones.

\section{Multi-step Cancer Development}

The six elements of the model of multi-step cancer development [2] including uncontrolled proliferation, growth suppressor inactivation, cell death resistance, replicative immortality, angiogenesis induction and acquisition of an invasive and metastatic phenotype should be considered when defining a novel therapeutic regimen to eradicate primary and metastatic disease. Furthermore, these elements are important considerations for successful cancer therapy in the context proposed in systems cancer medicine including predictive, preventive, personalized and participatory (P4) medicine [3]. This new paradigm in cancer medicine will transform medicine from a passive discipline (that responds only after the disease has progressed to a state that shows debilitating symptoms) to an active approach that focuses on ways to maintain individual wellness.

\section{Successful Cancer Therapy Requires Personalized Medicine}

Considering all these important issues, limited success in cancer therapy (in spite of the tremendous amount of data generated), is understandable given that cancer is not a single ailment, but rather a combination of multiple diseases with complexity and widespread heterogeneity within any single tumor type. This in turn mandates researchers to rethink strategies at both the basic and clinical levels to better understand the different aspects of cancer as a complex multidisease process and treat it accordingly. Given this perspective, the vision of completely curing cancer seems improbable, although not impossible. In order to overcome the challenges in getting there, a new approach based on personalized medicine should be the center of any novel anticancer strategy. In this context, both tumor and patientassociated variables, in combination with assessment of individualized response parameters, should be taken into account to adjust therapeutic regimens based on the patient's specific tumor characteristics [4].

\section{Complexity in Tumor Cells}

Complexity in tumor cells is a significant impediment toward understanding tumor biology that requires assimilating enormous amounts of genomic and proteomic data for each patient to guide successful clinical therapy. Indeed, it has been predicted [3] that by 2020 , billions of data points will exist (in a virtual cloud) for every single cancer patient. Therefore, we will need exceptionally powerful computational tools and necessarily concomitant bioinformatics expertise to de-convolute the complex dimensionality of this 
enormous patient-specific dataset into a manageable health diagnosis and disease status indication for each patient. The complexity of genomics profiling is readily evident in genotype-wide single nucleotide polymorphism (SNP) screening in predictive responses. It has been shown that patient SNPs can be used as prognostic markers for chemosensitivity to multimodal treatments [5].

\section{The Cancer Genome Atlas (TCGA) Project}

In an extensive attempt to unlock the complexity of tumor cells aberrations, The Cancer Genome Atlas (TCGA) project was launched several years ago as a joint effort of the National Cancer Institute (NCI) and the National Human Genome Research Institute (NHGRI). The focus of the TCGA project is on uncovering the molecular drivers of cancer through genome analysis, including large-scale genome sequencing, DNA copy number variation (CNV), methylation analysis, transcriptional profiling and assessment of splicing aberrations. The main goal of this rather ambitious project is to elucidate the entirety of the molecular and spatio-physical maps of cancer aberrations to improve the availability of cancer data for researchers and oncologists alike in an effort to aid diagnosis, treatment and prevention of cancer. In addition to TCGA project, the Genome Wide Associations Studies (GWAS) and various world-wide genomics studies are also attempting to map genetic aberrations and genetic susceptibility to cancer. This international effort underscores the challenges associated with understanding the impact of genetic network aberrations in tumor cells [6]. Overall, a multimodal omics approach including genomics, transcriptomics, methylomics, proteomics and metabolomics will comprehensively interrogate the patient tumor and allow researchers to learn important aspects of tumor initiation and progression to metastatic and drug resistant phenotypes. Along with technological advancement in omics, new computational tools are being developed to provide a powerful means to translate complex knowledge into daily clinical practice. Prior to the advent of polymerase chain reaction (PCR), oncologists were limited to information generated by a few molecular tests, conventional clinical presentation and histopathological analyses to choose among a small subset of available therapeutic regimens. Glioblastoma multiforme (GBM) was one of the first cancers studied by TCGA and this inquiry resulted in the discovery of genomic dysregulation of RB, $\mathrm{p}^{53}$ and RTK/RAS/PI3K as core biological pathways disrupted in GBM $[7,8]$.

\section{FDA Approval of Next Generation Sequencing Technologies}

The early promising results of genome sequencing data convinced authorities at the Food and Drug Administration (FDA) to approve marketing of Illumina's MiSeqDx as the first high-throughput (NextGen) genomic sequencer [9]. Next generation technology was the logical successor to groundbreaking, but limited (in terms of data mining capacity) cloning methods used in the early 90's to uncover genes such as the CFTR [10], NF-1 [11] and BRCA1 [12,13]. Advanced technologies such as the MiSeqDx [9], MI-ONCOSEQ [14-16] and Oncomine $[17,18]$ will allow oncology researchers and physicians to access genome-wide coverage, more accurately, faster and less expensively than prior methods. Furthermore, using an integrative high-throughput sequencing approach for patients with advanced cancer will generate a comprehensive mutational map that facilitates new clinical trials based on the identified biomarkers in patients' tumors. These are a few examples of the early successes and promise that genomic profiling and characterization holds. Similar findings are anticipated for other human cancers by employing current technologies as well as those presently under development to further explore complexity and heterogeneity of tumor cells, particularly at the single cell level.

\section{Single Cell Genomics in Cancer Prevention and Treatment}

The National Cancer Institute defines prevention as "the reduction of cancer mortality via reduction in the incidence of cancer". In this respect, both sets of factors including negative (associated with risk) and positive (with preventive effects) such as lifestyle, diet, smoking, alcohol consumption and preventing infections should be thoroughly investigated [19]. A dramatic drop in cancer incidence and death can only be expected by fully understanding the processes of carcinogenesis such as cancer biology and tumor metabolism using advanced technologies including genomics, transcriptomics, metabolomics and proteomics. Such an approach will definitely need to dovetail with chemoprevention, identification of individuals/ families with genetic predispositions and comprehensive information on biomarkers for early diagnosis of cancer. Thus far, even PSA screening for prostate cancer (one of the most widely used programs) has not been an entirely successful clinical tool [20,21]. In a genotype screen, the SNP309 T>G polymorphism, which is located in the promoter region of MDM2 gene, was identified and reported to contribute to the risk of colorectal In another study using whole transcriptome sequencing, a novel RUNX1-RUNX1T1 pathway was identified to be upregulated in clear cell renal cell carcinoma and considered an important factor contributing to the etiology of this cancer type [22]. Currently, success in cancer prevention suffers from incomplete information on etiology of all different human cancers and lack of accurate cancer susceptibility screening methods. Therefore, cancer preventive approaches are expected to benefit from advancement in genomic studies. Until then, new chemopreventive agents are in great demand to reduce cancer initiation, stop or delay tumor promotion and progression preferably by targeting tumor selfrenewal pathways. The use of anti-inflammatory agents such as aspirin, and later on, the anti-diabetic drug metformin has been associated with a decreased risk of cancer development. Interestingly, metformin has been found to target breast cancer stem cells (CSCs) in mouse models [23]. Furthermore, our group has shown that dietary compounds such as curcumin, found in turmeric [24], and sulforaphane, which is found in broccoli, can inhibit stem cell selfrenewal pathways and may prove useful for cancer prevention [25].

\section{Can Single Cell Studies Unravel Tumor Heterogeneity?}

Heterogeneity in cancer is mainly related to the genetic perturbations and the fact that random mutation frequency in human cancer cells is several hundred-fold greater than in adjacent normal cells [26]. Furthermore, epigenetic mechanisms such as DNAmethylation, histone-modification and non-coding RNA expression also significantly contribute to the complexity and heterogeneity of tumor cells. Molecular studies on seemingly homogenous population of tumor cells have revealed that single tumor cells can exhibit tremendous heterogeneity in gene expression, protein levels and phenotypic output that exerts crucial functional consequences $[27,28]$ In comparison to earlier studies of cellular heterogeneity that, due to technological issues, were limited to a few targeted RNAs or proteins, newer studies have benefitted from advancement in microfluidic 
methodologies for single cell analysis [29-31]. Researchers have used single cell RNA-Seq and RNA-Fluorescent in situ hybridization (FISH) methods to investigate cellular heterogeneity and observed bimodal variation in gene expression and splicing patterns that have not been previously reported. Surprisingly, this bimodal pattern in key immune genes across cells was also observed even for genes that are very highly expressed in the bulk population [32]. Although challenging, it is vitally important to compare single cell data sets from different studies with respect to the source of the analyzed samples, the biological status of the individual cells and the cellular microenvironment that highly influence the extent of single cell heterogeneity within a biological system. Studying single cell genomics may also help inform new schemes for cell characterization, identify cellular transition states, uncover hidden biological features and map molecular markers that can be used for new classifications of heretofore seemingly homogenous populations. To accomplish this goal, novel strategies are required to address the high level of noise inherent in single cell genomics related to technical difficulties of extremely low amounts of input material as well as biological issues such as transient bursts of RNA transcription [33]. Combining single cell studies and stochastic gene expression analyses can reveal both qualitative and quantitative characteristics of gene regulation that otherwise would remain hidden in population averaging gene expression studies [34]. In order to facilitate single cell studies, microfluidic devices are now being developed to do next generation sequencing (NGS) on appropriate linear mRNA after amplification and bar-coding, in much less time than prior generation of genome experiments.

\section{Novel Microfluidic Technologies to Study Breast CSCs Heterogeneity}

Collaborations between translational labs and biotechnology companies including Fluidigm Corporation (San Francisco, CA) and Denovo Sciences (Plymouth, MI) are underway for developing and/or optimizing microfluidic devices to study the heterogeneity of breast CSCs and circulating tumor cells (CTCs) at the single cell level. In a preliminary attempt to explore heterogeneity of CSCs and CTCs, our group has determined the gene expression signature of the CD44+/ CD24-, ALDH+ sorted CSC populations and bulk cells from breast cancer cell lines and patient derived xenografts at the single cell level (Figures 1 and 2) using Fluidigm's $\mathrm{C} 1$ and BioMark HD platforms. These three sorted fractions show distinct patterns of gene expression from one other, but also clearly show heterogeneity within each sorted population of CSCs. This observed heterogeneity would otherwise be obscured using conventional gene expression methods based on average population studies [35]. We and other researchers in the field believe that single cell analysis will soon become a transformational technology in cancer biology as well as in clinical cancer practice [36-38]. Future studies combining thousands of single cancer cells using these advanced technologies and others for assay preparations along with the novel computational methods will enable researchers to better reconstruct intracellular networks, re-evaluate cell types and states and transform our knowledge about the process of decisionmaking in individual cells at the genomic level.

\section{Hierarchical Model in Breast Cancer}

Breast cancer is ideal for studying tumor heterogeneity, because it is hierarchically organized, similar to the normal mammary epithelium and the disease process is known to be highly heterogeneous. The heterogeneity of breast cancer is manifested by its classification into a number of distinct subtypes, including luminal, HER2 positive, triple negative and claudin-low, featuring significantly different transcriptome and molecular expression signatures $[39,40]$.

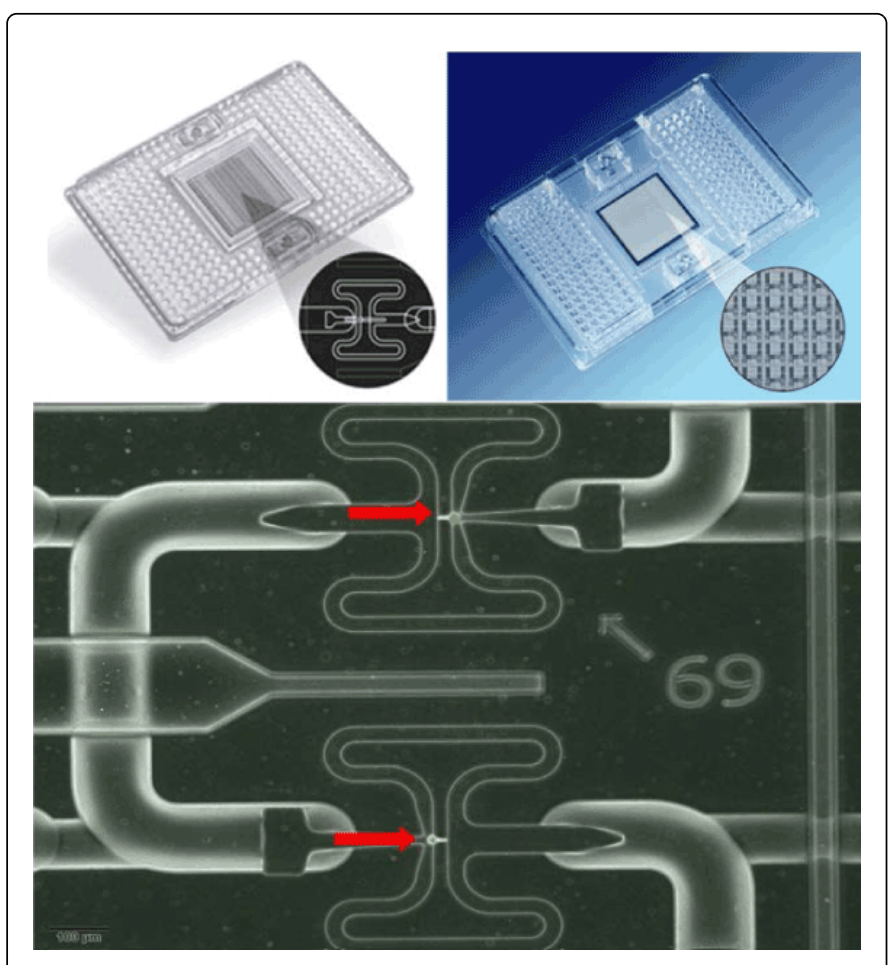

Figure 1: Representative image of the $\mathrm{Cl}^{\mathrm{m}}$ Single-Cell Auto Prep Array IFC for PreAmp containing 96 chambers for single cell isolation and pre-amplification of 96 target mRNA (top left), BioMark HD Dynamic Array IFCs to run 96x96 qRT-PCR assays (top right) and single cancer cells captured in chamber 69 and 70 (Green, ALDH+ cells) of the C1 Array IFC (bottom).

Our research and that of others has shown that hierarchically organized breast cancer is driven by a small fraction of tumor cells that display stem cell properties [41-43]. This small population of breast CSCs also termed breast cancer initiating cells was first identified among solid tumors by virtue of their expression of the cell surface markers $\mathrm{EpCAM}^{+} / \mathrm{CD} 24^{-} / \mathrm{CD} 44^{+}$and their capability to recapitulate heterogeneous populations of tumor cells [44]. Furthermore, it has been shown that both normal and malignant breast CSCs express high levels of the enzyme aldehyde dehydrogenase (ALDH) that serves as a predictor of poor clinical outcome in breast cancers [45]. These two types of breast CSCs are anatomically distinct, one with EMT (epithelial-to-mesenchymal transition) and one with MET (mesenchymal-to-epithelial transition) gene expression profiles. Furthermore, they dynamically show transition between the mesenchymal and epithelial-like states reflective of their normal counterparts in the mammary epithelial hierarchy [46]. Interestingly, this plasticity of breast CSCs from a quiescent mesenchymal state to a proliferative epithelial-like state plays a critical role for these cells to establish sizable metastatic nodules in distant organs. Indeed, there is increasing experimental evidence to suggest that such transition, termed colonization, is essential for development of successful macrometastasis [47]. 
Citation: Azizi E, Clouthier SG, Wicha MS (2014) The Promise of Single Cell Omics for Onco-therapy. J Mol Genet Med 8: 121. doi:
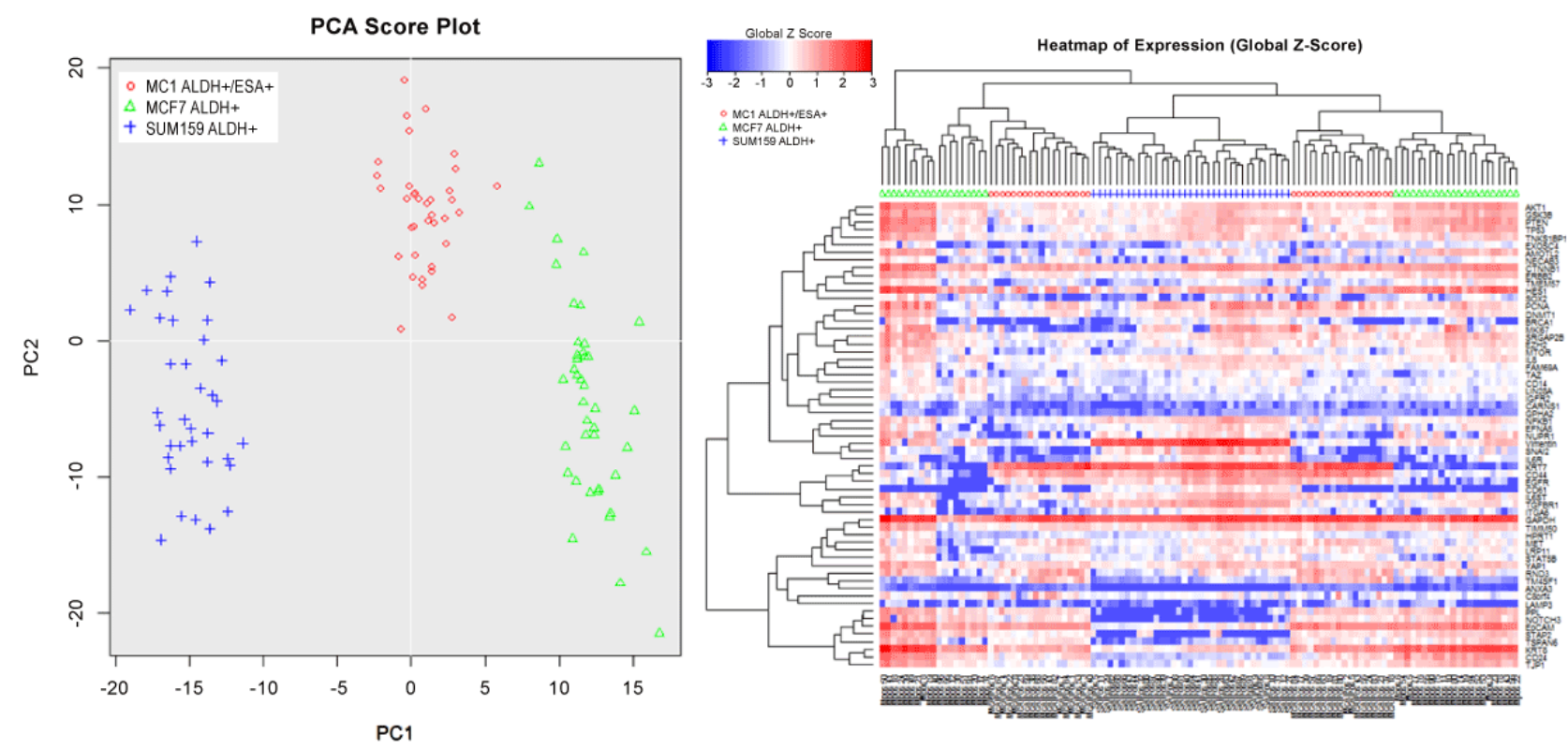

Violin Plot of Gene Expression By the Order of ANOVA P-Values

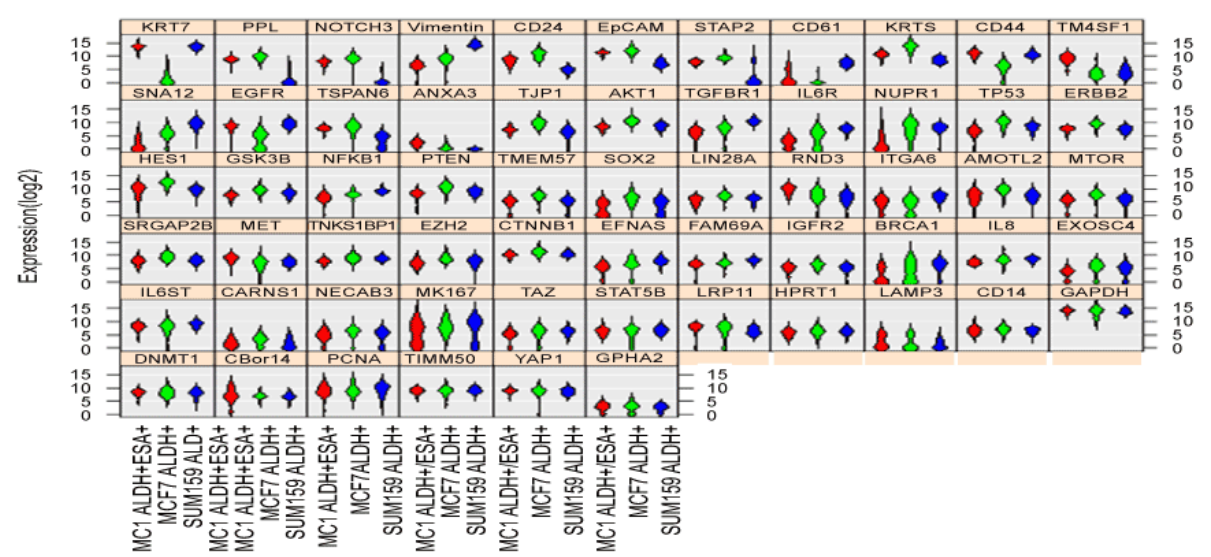

Figure 2: Single cell gene expression signature of the ALDH+ sorted CSC population of SUM159 (basal type; Blue) and MCF7 (luminal type; Green) breast cancer cell lines and MC1 (patient derived xenograft; Red); Data generated using Fluidigm's C1 and BioMark HD platforms and analyzed using Fluidigm's SINGuLAR software and R script and presented as log2Exp in principal component analysis (PCA; top left), Heat map clustering (top right) and Violin plot (bottom) formats.

\section{Importance of CTCs in Tumor Metastasis}

Metastasis, a major cause of cancer related death, starts with the dissemination of cancer cells from the primary site to the blood stream and ends with tumor formation in one or more distant organ sites. Cancer cells that enter the blood stream are called CTCs and are reportedly enriched in CSCs $[48,49]$. The majorities of the cells that enter the bloodstream from primary tumors are dead or dying as a consequence of shear force, apoptosis, necrosis or anoikis, or are directly eliminated by the immune system. Therefore, only a small fraction of CTCs survives to extravasate into distant sites. Those successfully disseminated tumor cells may grow to form a metastasis or may remain dormant for many years awaiting the appropriate microenvironmental cues to re-initiate their unchecked growth and differentiation program [50]. Although, most of the studies in the past focused on identifying CTCs of an epithelial phenotype [51-53], it has been increasingly recognized that CTCs are themselves a heterogeneous population of tumor cells consisting of several subpopulations with different characteristics such as CSCs and/or cells with the EMT phenotype that might be drivers of metastasis. Although epithelial cell adhesion molecule (EpCAM)-based systems are valuable tools for CTC enrichment, they lack comprehensive coverage to also isolate non-epithelial CTCs including EMT, dual epithelial/EMT, irreversible EMT and cancer stem cells. The inadequacy of EpCAMbased CTC capture methods, including CellSearch, underscores the importance of profiling the entire CTC population in order to develop the most robust and informative liquid biopsy [54]. 


\section{Towards a Liquid Biopsy to Interrogate Metastasis}

The prospect of a non-invasive liquid biopsy that could elucidate metastatic mechanisms makes CTCs an active area of cancer research. In addition, researchers have studied blood markers and identified transcripts that were organ-specific by deep comparative transcriptome analysis across forty or more different organs in humans and mice [3]. These data suggest that investigators will be able to assess early disease detection as well as disease progression through this non-invasive blood monitoring approach. This methodology of organ-specific blood fingerprinting will enable oncologists to more readily distinguish disease status, assess disease progression, monitor response to therapy and better determine probabilities for cancer recurrences. In addition to CTCs and blood proteins as tumor biomarkers, circulating DNAs, mRNAs and microRNAs from tumor cells are being studied as surrogate tumor biomarkers and for monitoring cancer recurrence [55-58]. In addition, accumulating evidence suggest that CTCs may display phenotypes distinct from their corresponding primary tumors [59-61]. This discordance may reflect tumor evolution as well as differential expression of markers on CSCs and bulk tumor cell populations. Very recently, our laboratory in collaboration with the BioMEMS for CTC research laboratory at the University of Michigan has jointly characterized isolated CTCs by using a highly-sensitive microfluidic capture device (Figure 3) and found HER2 positive CTCs from the blood of metastatic breast cancer patients had HER2 negative primary tumors [62]. This provides a potential explanation for the surprising finding that HER2 blockade in the adjuvant setting benefits women whose breast tumors do not display HER2 gene amplification. In addition, in a study on prostate cancer patients, researchers examined the functional diversity of viable, single CTCs for clonal comparison and mapping of heterogeneity. They reported that only a rare subset of isolated CTCs were resistant to anoikis within blood circulation, showing metastatic features such as invasiveness and producing proteases in patients with late-stage, metastatic castration-resistant prostate cancer (mCRPC). These data further suggest that enumeration of CTCs alone may be insufficient to fully interrogate the metastatic potential of tumor cells in the circulation of cancer patients [63]. Furthermore, disseminated tumor cells (DTCs) in the bone marrow of breast cancer patients have also been studied in tumor metastasis.

\section{Novel Genomic Methods in Studying CTCs and DTCs}

In addition to CTCs, DTCs from breast cancer patients have also been studied as an independent prognostic factor using whole-genome amplification (WGA) followed by NGS and reported a clear difference in the copy number between the DTCs and matched primary tumors, indicating that the DTC underwent further evolution at the copy number level $[64,65]$. It has also been reported that the PCR-based WGA methods may better preserve single cell DNA copy number changes during the amplification process and may also be employed for single-nucleotide variant detection [31]. Another improvement in technologies related to single cell analysis is development and validation of a novel and robust mRNA-Seq protocol (Smart-Seq) that has improved read coverage across transcripts to be able to significantly enhance detailed analyses of alternate transcript isoforms and better identify SNPs. Researchers have recently used this sensitive and quantitative technique on single melanoma CTCs and identified distinct gene expression patterns, including new candidate biomarkers for melanoma CTCs [31].

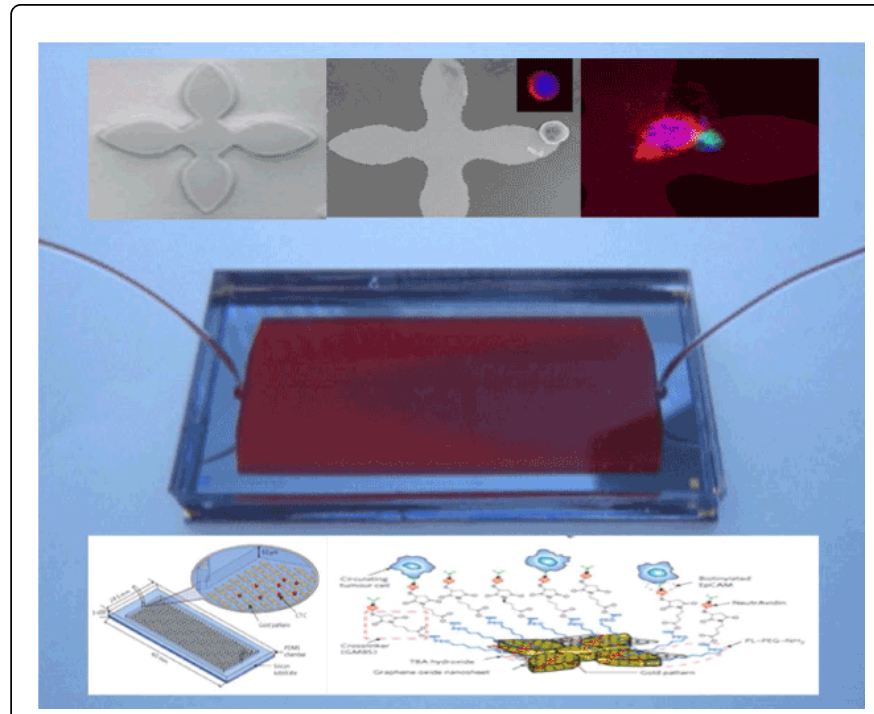

Figure 3: Representative images of star shape capture post before (top left) and after (top middle) capturing single CTC. Captured CTCs alone (top middle) or bound to WBC (top right) stained with antibodies against panCK (Red), CD45 (Green) and DAPI (Blue). Graphene Oxide (GO) microfluidic device filled with a patient's blood sample (middle). Schematic representation of the functionalized GO device (bottom) that is highly sensitive to capture CTCs.

Therefore, single cell analyses of CTCs and DTCs are an important tool for exploring tumor heterogeneity as well as complexity of the cancer genome [65-67]. Blood is anticipated to be utilized as the preferred patient sample in the clinic in the near term as a window for monitoring tumor progression and response to therapy. The attraction for researchers to use microfluidic technologies along with the omics tools to further explore CTCs is the non-invasive nature of liquid compared to traditional tissue biopsy protocols. Technical improvements in CTC collection and characterization will lead to advancements in the liquid biopsy approach that will ultimately guide oncologists to select more successful anti-cancer therapies based on both tumor cells characteristics and individual patient response. Furthermore, single cell genomic studies on CTCs (as well as those on primary and metastatic tumor cells) will have a profound impact on cancer preventative approaches for tumor initiation and/or progression to drug resistant and metastatic phenotypes.

\section{Tumor Cell Genome Analysis for Cancer Therapeutics}

Genomic analysis is expected to untangle the complex nature of cancer biology and the extensive tumor heterogeneity leading to identification of new targets for more successful cancer therapy [68] Current anticancer regimens mostly target the rapidly proliferating bulk tumor cells to shrink the tumor mass. Despite effectiveness of these therapeutic strategies in reducing the size of primary tumors, they frequently fail to eradicate advanced tumors and are associated with tumor relapse. The CSC hypothesis suggests that conventional anti-tumor strategies targeting rapidly proliferating cells may fail to target CSCs that divide less frequently in the tumor [69]. A plethora of studies have shown that BCSCs are relatively resistant to both ionizing radiation and chemotherapy [70-73]. Genomic and other molecular 
studies on the pathways that regulate CSC self-renewal and survival have revealed numerous new targets for therapeutic development. Indeed, several biotechnology and pharmaceutical companies are currently developing agents to target CSCs. These agents commonly target the Notch, Hedgehog, Wnt, Akt/mTOR and NFkB signal transduction pathways. Some of these targeted agents are already in early phase trial awaiting phase II/III assessments to determine clinical efficacy $[69,74]$. In the context of developing new agents to target CSCs, genomic analysis will provide crucial insight into prediction of the rate of success and in control or eradication of heterogenous tumor cells. For example, in a genome-wide SNP screening, patients with tumors that were homozygous for the wild type alleles of LIFR rs3729740 treated with Cetuximab and ANXA11 rs1049550 in patients treated with Bevacizumab showed some degree of predictive potentials for chemoresistance to these targeted therapeutic agents [5].

\section{Omics Studies in Other Cancer Types}

Whole genome mapping of promoters that are activated by DNA hypomethylation has been used in hepatocellular carcinoma (HCC). Gene expression inhibition by siRNA or shRNA reduced human tumor xenograft growth in mice and altered other tumor characteristics in, breast and bladdercell lines. Results indicate that RASAL2 (a tumor and metastasis suppressor) and NENF (a neuronderived neurotrophic factor) depletion interferes with Akt, Wnt and MAPK signaling pathways as well as regulation of epigenetic drivers ofgrowth and metastasis [75]. In addition, a new NGS-based method to identify somatic mutations has been used to do comprehensive mutation profiling in thirty genes found frequently mutated in lung adenocarcinoma. The variations in TP53, KRAS, STK11 and EGFR were detected in lung cancer cells that were also previously reported as somatic mutations in the COSMIC database, with more than $1000 \times$ coverage. These mutation profiles were validated by DNA microarray-based genotyping and indicated high-throughput mutation profiling is a robust and effective tool for somatic variant screening [76]. A targeted sequencing platform using NGS technology has been established for clinical use in colorectal cancer to explore mutation and $\mathrm{CNV}$ data. A total of 526 somatic non-synonymous sequence variations were found in 113 genes such as APC, TP53 and KRAS. This study revealed ErbB signaling pathway as the most commonly involved pathway [77]. Sequencing techniques were also used to evaluate mutation profile of telomerase reverse transcriptase (TERT) and telomerase RNA component (TERC) in 143 esophageal cancer patients. This study revealed one deletion in TERC and two non-synonymous variants in TERT and down-regulation of canonical Wnt signaling in cancer cells [78]. In order to better understand cellular pathways and facilitate drug discovery, a project named the Library of Integrated Network-Based Cellular Signatures (LINCS) has been established to look at genomics and proteomics alterations associated with cell perturbations following exposure to different compounds. Furthermore, a novel binary linear programming (BLP) was developed and applied to the MCF7 breast cancer cell line to study signaling response of phosphorylation. This approach was also applied to the PC3 prostatecell line and the cross-validation analysis showed a high level of accuracy in predicting effects of test compounds [79].

\section{Genome Sequencing and the Development of Novel Therapeutic Targets}

As a result of genomic sequencing studies, two novel smallmolecule drugs, crizotinib and PLX4032, have been advanced to late phase clinical trials for their impressive anticancer effects on NSCLCs carrying EML4-ALK translocations $[80,81]$ and metastatic malignant melanomas carrying the V600E mutation of the $B R A F$ gene [82]. Additionally, it has been shown in a mouse model that stimulatory effects of sympathetic activation on bone metastasis of breast cancer cells can be blocked by beta blockers and or inhibition of RANKL signaling [83]. Recent research has revealed an ETS fusion in prostate cancer cell lines and mouse xenograft models and reported that PARP1 inhibition can sensitize tumor cells to radiation therapy by contracting ERG/PARP enhanced DNA repair activities. [84]. This same group studied micropapillary carcinoma (MPC), a histologically rare but aggressive type of breast cancer, using the Sequenom OncoCarta mutation analysis and RNASeq methodology to identify expressed fusion genes [85]. In this study, two in-frame fusion genes, SLC2A1-FAF1 and BCAS4-AURKA, with stimulatory effects on cell proliferation were identified in both primary and lymph-node metastasis samples. In addition, they reported that disruption in the CDK12 gene as a result of somatic rearrangement observed in the subset of HER2-amplified breast cancers can sensitize these tumor cells to PARP inhibition. This finding provides molecular rational for therapeutic benefits of PARP inhibitors in treatment of the HER2 positive cancers. Another group of researchers used a proteomics rather than genomics approach to study the impact of ERBB2/ HER2 amplification that activates signaling cascades in breast CSCs. They reported that protein content of extracellular vesicles was relevant to cellular malignancy and therefore can be potentially exploited as biomarker for HER2 ${ }^{+}$cancer patients [86].

\section{First Successful Targeted Therapy in Breast Cancer}

Trastuzumab, an anti-HER2 antibody, was the first successful targeted therapy available for treatment of HER2-positive breast cancers. Current clinical practice dictates anti-HER2 therapy should only benefit to breast cancer patients with HER2 positive primary tumors. Recently our group demonstrated that increased HER2 expression, but not HER2 gene amplification, in the breast CSCs is mediated by the receptor activator of NF- $\kappa \mathrm{B}$ (RANK)-ligand in the bone microenvironment. Therefore, patients with HER2 negative luminal breast cancers may benefit from adjuvant Trastuzumab therapy to target breast CSC population $[87,88]$. Unfortunately, resistance to Trastuzumab in some HER2 positive breast cancers resulted in incurable metastatic disease $[89,90]$. One of the possible reasons for development of Trastuzumab-resistance has been explored in the PTEN knockdown breast cancer cell lines and in mouse xenograft models. It has been demonstrated that an inflammatory feedback loop mediated by IL6 in the absence of PTEN is responsible for the development of Trastuzumab resistance in breast CSCs.

\section{Inflammatory Interleukins in Trastuzumab Resistance}

The inflammatory feedback loop can be inhibited by an IL6 receptor antibody [91]. Furthermore, the role of IL8 in regulating the breast CSCs has been reported to be partly due to a novel SRC and EGFR/HER2-dependent pathway [92]. These researchers were able to demonstrate that in HER2-positive breast cancers, IL8 blocking effect on mammosphere formation via CXCR $1 / 2$ inhibition increases the efficacy of Lapatinib, a dual inhibitor for EGFR/HER2 tyrosine kinase. These data suggest that combination of HER2 targeted therapies with the CXCR1/2 inhibitors may improve the survival of HER2-positive patients via targeting breast CSCs. In addition, the combination of 
Lapatinib with chemotherapy showed a decrease in time to progression of trastuzumab-resistant patients [93,94].

\section{Anti-Notch Targeted Therapy}

Other molecular studies on cancer cells found that inhibiting the Notch signaling pathway is potentially promising in targeted CSCs therapy $[74,95]$. Therefore, researchers have investigated the effects of small molecules with $\gamma$-secretase inhibitory activities or monoclonal antibodies against Notch ligands and/or receptors that resulted in a few promising agents that are currently in clinical development. In addition to targeted therapy against CSCs and chemotherapy, antihormone therapy is another important choice in hormone-dependent malignancies such as breast and prostate cancers.

\section{Single Cell Genomics in Hormonal Therapy}

Recently a group of researchers characterized the single cells and expression signatures of hormone-starved MCF-7 cells that respond to estrogen [96]. In this study, dissecting single cell states from timecourse microarray data revealed that within less than two days following stimulation, MCF-7 cells transit through six different cellular states. Furthermore, they employed genome-wide transcriptional profiling of single cell states and also functional characterization that supported a scenario of promoting estrogen effects on cell cycle progression. Employing this method also enabled researchers to identify estrogen target genes involved in each of the six transitional states. Combining these data with the findings of another study [97] on the efficacy of anti-estrogen drugs such as ICI 182,780/ Faslodex, a pure anti-hormone used in hormone therapy of hormoneresponsive breast tumors, provides a genetic explanation for dynamic responses of breast cancer cells to anti-hormone therapy. Together these results describe the impact of mutational perturbations of one or more single cell states resulting in cell cycle progression to a hormoneindependent manner. Furthermore, these findings explain one of the main reasons for development of anti-hormone resistant phenotype in about $30 \%$ of anti-hormone treated breast tumors.

\section{Challenges in Single Cell Genomic Studies}

Although genome analyses at the single cell level can resolve ambiguities associated with data generated from average population, such analyses are error prone due to WGA artifacts and are also limited in the types of identifiable DNA mutations $[98,99]$. Researchers have been able to develop methods for paired-end sequence analysis of single cell WGA products to detect multiple classes of DNA mutation, discriminate the CNVs from allelic WGA artifacts and specify the break points and architecture of structural variants [100]. Furthermore, the NGS approach for DNA analysis provides potential advantages in comparison to the traditional methods, including the ability to fully sequence thousands of genes in a single run. NGS can also simultaneously identify multiple genetic aberrations including insertions and deletions, CNVs, translocations and many more genetic perturbations. However, important challenges, particularly with respect to demands on expertise and infrastructure, need to be addressed in order to translate data generated by NGS to make it "clinically-actionable" [68]. Highly anticipated new technologies, far better than NGS and WGS, will soon emerge with clinically preferred features such as reduced overall cost, faster turnaround time, more genome coverage as well as epigenome interrogation, to be applied on ever-minute amounts of specimens including single CTCs and circulating free DNAs, RNAs and proteins in blood samples of cancer patients.

\section{New Advancements in Single Cell Proteomics}

New advancements in multiplexing and high-throughput assays for proteomic studies have been innovated by using mass cytometry to determine the expression and abundance of key target proteins under conditions of interest. The DVS Sciences mass cytometry technology, CyTOF, (recently acquired by Fluidigm Corporation) and similarly developed technologies allow cancer researchers to study several target proteins simultaneously in each single cancer cell. In mass cytometry, rare earth metals are used in conjugate with antibodies that allows determination of protein markers expression in individual cells based on the metal abundances. This highly advanced methodology completes the chain of data generation on tumor heterogeneity from genome to proteome characterizations of single cancer cells $[101,102]$. Mass cytometry has previously been applied only to cell suspensions but to gain spatial information, researchers have coupled immunohistochemical and immunocytochemical methods with highresolution laser ablation to $\mathrm{C}_{\mathrm{y}} \mathrm{TOF}$ mass cytometry. Simultaneous imaging of thirty-two proteins and protein modifications has been achieved at subcellular resolution that is expected to be increased to one hundred markers using additional isotopes. Researchers have applied imaging mass cytometry to human breast cancer samples, to explore subpopulations of cancer cells and cell-cell interactions for highlighting tumor heterogeneity. Mass cytometry will enable researchers to study heterogeneity and function of cancer cells and further support the transition of medicine toward individualized molecularly targeted diagnosis and therapies [103].

\section{Newer Single Cell Omics Technologies}

Newer omics technologies including metabolomics [104] and methylomics $[105,106]$ have emerged and been used at both the bulk and single cell level to further explore tumor heterogeneity and complexity with respect to metabolic status (as a measure of dynamic functionality) and DNA methylation patterns (controlling gene expression and phenotypic characteristics). Researchers have used metabolomics approach in metastatic androgen-dependent prostate cancer (AD) and castration-resistant prostate cancer (CRPC) cell lines to measure levels and utilization rate of metabolites using a combination of targeted mass spectrometry and metabolic phenotyping. Oncomine concept map (OCM) analysis revealed that the most altered metabolites in the CRPC in comparison to AD cells were related to biochemical pathways involved in the activation of UDP glucuronosyltransferase (UGT). In addition, data analysis of this study showed an association between the time to treatment failure that can potentially be used as a predictive marker in clinical settings for cancer therapy [107]. The latest set of metabolomics data combines GWA with high-throughput metabolic profiling to explore influences of genetic variation on metabolism in complex diseases. This study revealed important information on more than one hundred metabolic loci and their biochemically connected metabolites in human blood that is now available as database and web-based resources for the research community. These findings will provide new insights into the role of genetic variation in blood metabolic diversity that can be used to better understand the complexity of various disease processes including cancer and ultimately identify potential new targets for drug development [108]. Researchers have also employed whole DNA methylome (WDM) profiling to study the process involved in 
transition of lung cancer cells to a metastatic phenotype. Results of this study showed that the expression of several DNA methyltransferases and TET1 genes was altered after the EMT in the lung cancer cells. However, this TGF- $\beta$-induced EMT was not associated with the WDM alteration in cancer cells [109].

\section{Clinical Requirements of Omics-based Technologies}

Advancement in omics technologies and the generation of voluminous amounts of data on heterogeneity and complexity of cancer cells will necessitate a close cooperation between industry and academia to foster early stage drug discovery, biomarker characterization, unique animal model generation, target/pathway validation and clinical trial translational studies. Importantly, dual or multiple pathway inhibitors will be at a significant advantage since many cancers are heterogeneous and are driven by multiple cellular regulators. In addition, novel identification, isolation and molecular/ functional characterization assays are desperately needed for highly sensitive discrimination and quantification of CSCs in the primary and metastatic tumors as well as in the CTCs. One important clinical consideration is that stem cell targeting therapy would be much more beneficial if co-administered with conventional de-bulking anticancer agents to significantly increase the rate of successful oncology therapy. Overall, cancer genomics studies will provide information for clinical applications including new targeted anticancer drug development and screening tests to identify patients for being treated with such newly developed targeted therapies. The principle challenge now for cancer omics studies is to address remaining methodological hurdles with respect to increased genome coverage, assay speed and overall robustness. Furthermore, for clinical application of genomics data in daily practice, cost effectiveness for cancer patients and insurance plans as well as simplifying bioinformatics data for oncologist are important issues that must be adequately addressed.

\section{Conclusion}

The clinical efficacy of cancer therapies has been limited by tumor complexity, heterogeneity and resistance mechanisms inherent in tumor clones. Revolutionary single cell technologies will allow researchers to study omics of cancer cells to unlock these unknown, but important features of different tumors. This in turn is expected to improve several aspects of cancer medicine, including early detection, monitoring CTCs, assessing tumor cells heterogeneity and guiding anticancer therapy that are crucial for successful cancer treatment. Therefore, the clinical value of omics studies at the single cell level will be most evident with improved profiling of scarce CTCs and CSCs in clinical samples and detecting clones with drug resistant and metastatic features. In addition to advancement in single cell omics technologies, adopting these methods to clinical daily practice is desperately needed. The accuracy and speed in generating valuable patient-specific omics data in a more streamlined manner for use by researchers and oncologists alike should be considered as important as the cost for cancer diagnosis and treatment.

\section{Acknowledgements}

The authors would like to acknowledge valuable scientific collaborations with Dr. Sunitha Nagrath, head of BioMEMS lab at UM and her lab members for the development of microfluidic devices necessary to isolate pure CTCs from whole blood, Shamileh Fouladdel for conducting single cell gene expression experiments using
Fluidigm's C1 and BioMark HD instruments, Sean McDermott and Rachel Martin for conducting murine xenograft experiments and Hui Jiang and Mary Sehl for BioInformatics data analysis. The authors would also like to express gratitude to senior management of both Fluidigm Corporation and Denovo Sciences for working closely with our laboratory personnel to develop microfluidic platforms to accurately capture and profile CTCs and CSCs from breast cancer patient sample. In addition we thank Denise Poirier and Courtney LeBlanc for invaluable assistance preparing the manuscript.

\section{Disclosure}

MSW has financial holding and is a scientific advisor for OncoMed Pharmaceuticals, is a scientific advisor for Verastem, Paganini and MedImmune and receives research support from Dompe Pharmaceuticals and MedImmune. EA and SGC have no competing financial relationships to disclose.

\section{References}

1. Cohen N, Kravchenko-Balasha N, Klein S, Levitzki A (2013) Heterogeneity of gene expression in murine squamous cell carcinoma development-the same tumor by different means. PLoS One 8: e57748.

2. Hanahan D, Weinberg RA (2011) Hallmarks of cancer: the nextgeneration. Cell 144: 646-674.

3. Tian Q, Price ND, Hood L (2012) Systems cancer medicine: towards realization of predictive, preventive, personalized and participatory (P4) medicine. J Intern Med 271: 111-121.

4. Brücher BL, Lyman G, van Hillegersberg R, Pollock RE, Lordick F, et al. (2014) Imagine a world without cancer. BMC Cancer 14: 186.

5. Kim JC, Kim SY, Cho DH, Ha YJ, Choi EY, et al. (2011) Novel chemosensitive single-nucleotide polymorphism markers to targeted regimens in metastatic colorectal cancer. Clin Cancer Res 17: 1200-1209.

6. Gonzalez-Angulo AM, Hennessy BT, Mills GB (2010) Future of personalized medicine in oncology: a systems biology approach. J Clin Oncol 28: 2777-2783.

7. Parsons DW, Jones S, Zhang X, Lin JC, Leary RJ, et al. (2008) An integrated genomic analysis of human glioblastoma multiforme. Science 321: 1807-1812.

8. Cancer Genome Atlas Research N (2008) Comprehensive genomic characterization defines human glioblastoma genes and core pathways. Nature 455: 1061-1068.

9. Collins FS, Hamburg MA (2013) First FDA authorization for nextgeneration sequencer. N Engl J Med 369: 2369-2371.

10. Riordan JR, Rommens JM, Kerem B, Alon N, Rozmahel R, et al. (1989) Identification of the cystic fibrosis gene: cloning and characterization of complementary DNA. Science 245: 1066-1073

11. Rosenfeld PJ, Kelly TJ (1986) Purification of nuclear factor I by DNA recognition site affinity chromatography. J Biol Chem 261: 1398-1408.

12. Newman B, Austin MA, Lee M, King MC (1988) Inheritance of human breast cancer: evidence for autosomal dominant transmission in high-risk families. Proc Natl Acad Sci U S A 85: 3044-3048.

13. Hall JM, Lee MK, Newman B, Morrow JE, Anderson LA, et al. (1990) Linkage of early-onset familial breast cancer to chromosome 17q21. Science 250: 1684-1689. 
14. Robinson DR, Wu YM, Kalyana-Sundaram S, Cao X, Lonigro RJ, et al. (2013) Identification of recurrent NAB2-STAT6 gene fusions in solitary fibrous tumor by integrative sequencing. Nat Genet 45: 180-185.

15. Roychowdhury S, Iyer MK, Robinson DR, Lonigro RJ, Wu YM, et al. (2011) Personalized oncology through integrative high-throughput sequencing: a pilot study. Sci Transl Med 3: 111.

16. Everett JN, Gustafson SL, Raymond VM (2014) Traditional roles in a non-traditional setting: genetic counseling in precision oncology. See comment in PubMed Commons below J Genet Couns 23: 655-660.

17. Rhodes DR, Kalyana-Sundaram S, Mahavisno V, Varambally R, Yu J, et al. (2007) Oncomine 3.0: genes, pathways, and networks in a collection of 18,000 cancer gene expression profiles. Neoplasia 9: 166-180.

18. Monami G, Emiliozzi V, Bitto A, Lovat F, Xu SQ, et al. (2009) Proepithelin regulates prostate cancer cell biology by promoting cell growth, migration, and anchorage-independent growth. Am J Pathol 174: 1037-1047.

19. Parkin DM (2006) The global health burden of infection-associated cancers in the year 2002. Int J Cancer 118: 3030-3044.

20. Xia J, Gulati R, Au M, Gore JL, Lin DW, et al. (2013) Effects of screening on radical prostatectomy efficacy: the prostate cancer intervention versus observation trial. J Natl Cancer Inst 105: 546-550.

21. Wang W, Du M, Gu D, Zhu L, Chu H, et al. (2014) MDM2 SNP309 polymorphism is associated with colorectal cancer risk. Sci Rep 4: 4851

22. Xiong Z, Yu H, Ding Y, Feng C, Wei H, et al. (2014) RNA sequencing reveals upregulation of RUNX1-RUNX1T1 gene signatures in clear cell renal cell carcinoma. Biomed Res Int 2014: 450621

23. Song CW, Lee H, Dings RP, Williams B, Powers J, et al. (2012) Metformin kills and radiosensitizes cancer cells and preferentially kills cancer stem cells. Sci Rep 2: 362 .

24. Kakarala M, Brenner DE, Korkaya H, Cheng C, Tazi K, et al. (2010) Targeting breast stem cells with the cancer preventive compounds curcumin and piperine. Breast cancer research and treatment 122: 777-785.

25. Li Y, Zhang T, Korkaya H, Liu S, Lee HF, et al. (2010) Sulforaphane, a dietary component of broccoli/broccoli sprouts, inhibits breast cancer stem cells. Clin Cancer Res 16: 2580-2590.

26. Raj A, van Oudenaarden A (2009) Single-molecule approaches to stochastic gene expression. Annu Rev Biophys 38: 255-270.

27. Kalisky T, Blainey P, Quake SR (2011) Genomic analysis at the single-cell level. Annu Rev Genet 45: 431-445.

28. Cohen AA, Geva-Zatorsky N, Eden E, Frenkel-Morgenstern M, Issaeva I, et al. (2008) Dynamic proteomics of individual cancer cells in response to a drug. Science 322: 1511-1516.

29. Tang F, Barbacioru C, Wang Y, Nordman E, Lee C, et al. (2009) mRNASeq whole-transcriptome analysis of a single cell. Nat Methods 6: 377-382.

30. Islam S, Kjällquist U, Moliner A, Zajac P, Fan JB, et al. (2011) Characterization of the single-cell transcriptional landscape by highly multiplex RNA-seq. Genome Res 21: 1160-1167.

31. Ramsköld D, Luo S, Wang YC, Li R, Deng Q, et al. (2012) Full-length mRNA-Seq from single-cell levels of RNA and individual circulating tumor cells. Nat Biotechnol 30: 777-782.

32. Shalek AK, Satija R, Adiconis X, Gertner RS, Gaublomme JT, et al. (2013) Single-cell transcriptomics reveals bimodality in expression and splicing in immune cells. See comment in PubMed Commons below Nature 498: 236-240.

33. Cai L, Dalal CK, Elowitz MB (2008) Frequency-modulated nuclear localization bursts coordinate gene regulation. Nature 455: 485-490.

34. Munsky B, Neuert G, van Oudenaarden A (2012) Using gene expression noise to understand gene regulation. Science 336: 183-187.

35. Azizi E, Fouladdel S, Deol YS, Bender J, McDermott SP, et al. (2014) Exploring cancer stem cells heterogeneity via single cell multiplex gene expression analysisAACR, San Diego.

36. Li G, Teng L (2014) A superior strategy for single-cell mutational screening via multiplex-targeted QPCR using the BioMark HD microfluidic platform. Future Oncol 10: 507-510.

37. Chen CL, Mahalingam D, Osmulski P, Jadhav RR, Wang CM, et al. (2013) Single-cell analysis of circulating tumor cells identifies cumulative expression patterns of EMT-related genes in metastatic prostate cancer. The Prostate 73: 813-826.

38. Livak KJ, Wills QF, Tipping AJ, Datta K, Mittal R, et al. (2013) Methods for qPCR gene expression profiling applied to 1440 lymphoblastoid single cells. Methods 59: 71-79.

39. Visvader JE (2009) Keeping abreast of the mammary epithelial hierarchy and breast tumorigenesis. Genes Dev 23: 2563-2577.

40. Cancer Genome Atlas Network (2012) Comprehensive molecular portraits of human breast tumours. Nature 490: 61-70.

41. Wicha MS, Liu S, Dontu G (2006) Cancer stem cells: an old idea--a paradigm shift Cancer Res 66: 1883-1890.

42. Liu S, Wicha MS (2010) Targeting breast cancer stem cells J Clin Oncol 28: 4006-4012.

43. Charafe-Jauffret E, Monville F, Ginestier C, Dontu G, Birnbaum D, et al. (2008) Cancer stem cells in breast: current opinion and future challenges Pathobiology 75: 75-84.

44. Al-Hajj M, Wicha MS, Benito-Hernandez A, Morrison SJ, Clarke MF (2003) Prospective identification of tumorigenic breast cancer cells Proc Natl Acad Sci U S A 100: 3983-3988.

45. Ginestier C, Hur MH, Charafe-Jauffret E, Monville F, Dutcher J, et al. (2007) ALDH1 is a marker of normal and malignant human mammary stem cells and a predictor of poor clinical outcome Cell Stem Cell 1 : 555-567.

46. Liu S, Cong Y, Wang D, Sun Y, Deng L, et al. (2014) Breast Cancer Stem Cells Transition between Epithelial and Mesenchymal States Reflective of their Normal Counterparts. Stem Cell Reports 2: 78-91.

47. Brabletz T (2012) To differentiate or not--routes towards metastasisNat Rev Cancer 12: 425-436.

48. Murray NP, Reyes E, Tapia P, Badinez L, Orellana N, et al. (2012) Redefining micrometastasis in prostate cancer - a comparison of circulating prostate cells, bone marrow disseminated tumor cells and micrometastasis: Implications in determining local or systemic treatment for biochemical failure after radical prostatectomy Int J Mol Med 30: 896-904

49. Lianidou ES, Markou A, Strati A (2012) Molecular characterization of circulating tumor cells in breast cancer: challenges and promises for individualized cancer treatment. Cancer Metastasis Rev 31: 663-671.

50. Joosse SA, Pantel K (2013) Biologic challenges in the detection of circulating tumor cells Cancer Res 73: 8-11.

51. Cristofanilli M, Hayes DF, Budd GT, Ellis MJ, Stopeck A, et al. (2005) Circulating tumor cells: a novel prognostic factor for newly diagnosed metastatic breast cancer. J Clin Oncol 23: 1420-1430. 
Page 10 of 11

52. Maheswaran S, Sequist LV, Nagrath S, Ulkus L, Brannigan B, et al. (2008) Detection of mutations in EGFR in circulating lung-cancer cells $\mathrm{N}$ Engl J Med 359: 366-377.

53. Nagrath S, Sequist LV, Maheswaran S, Bell DW, Irimia D, et al. (2007) Isolation of rare circulating tumour cells in cancer patients by microchip technology Nature 450: 1235-1239.

54. Grover PK, Cummins AG, Price TJ, Roberts-Thomson IC, Hardingham JE (2014) Circulating tumour cells: the evolving concept and the inadequacy of their enrichment by EpCAM-based methodology for basic and clinical cancer research. Ann Oncol.

55. Kim K, Shin DG, Park MK, Baik SH, Kim TH, et al. (2014) Circulating cell-free DNA as a promising biomarker in patients with gastric cancer: diagnostic validity and significant reduction of cfDNA after surgical resection. Annals of surgical treatment and research 86: 136-142.

56. Tsujiura M, Ichikawa D, Konishi H, Komatsu S, Shiozaki A, et al. (2014) Liquid biopsy of gastric cancer patients: Circulating tumor cells and cellfree nucleic acids. World journal of gastroenterology 20: 3265-3286.

57. Wang K, Zhang S, Marzolf B, Troisch P, Brightman A, et al. (2009) Circulating microRNAs, potential biomarkers for drug-induced liver injury Proc Natl Acad Sci USA 106: 4402-4407.

58. Lin EH, Hassan M, Li Y, Zhao H, Nooka A, et al. (2007) Elevated circulating endothelial progenitor marker CD133 messenger RNA levels predict colon cancer recurrence Cancer 110: 534-542.

59. Markiewicz A, Ksiazkiewicz M, Welnicka-Jaskiewicz M, Seroczynska B, Skokowski J, et al. (2014) Mesenchymal phenotype of CTC-enriched blood fraction and lymph node metastasis formation potential PLoS One 9: 93901.

60. Campos M, Luque R, Jiménez J, Martínez R, Warleta F, et al. (2013) Simultaneous phenotypic and genetic characterization of single circulating tumor cells from colon cancer patientsHistol Histopathol 28: 1439-1450.

61. Pailler E, Adam J, Barthélémy A, Oulhen M, Auger N, et al. (2013) Detection of circulating tumor cells harboring a unique ALK rearrangement in ALK-positive non-small-cell lung cancer J Clin Oncol 31: 2273-2281.

62. Yoon HJ, Kim TH, Zhang Z, Azizi E, Pham TM, et al. (2013) Sensitive capture of circulating tumour cells by functionalized graphene oxide nanosheets Nat Nanotechnol 8: 735-741.

63. Yao X, Choudhury AD, Yamanaka YJ, Adalsteinsson VA, Gierahn TM, et al. (2014) Functional analysis of single cells identifies a rare subset of circulating tumor cells with malignant traitsIntegr Biol (Camb) 6: 388-398.

64. Gruber I, Fehm T, Taran FA, Wallwiener M, Hahn M, et al. (2014) Disseminated tumor cells as a monitoring tool for adjuvant therapy in patients with primary breast cancer Breast Cancer Res Treat 144: 353-360.

65. Møller EK, Kumar P, Voet T, Peterson A, Van Loo P, et al. (2013) Nextgeneration sequencing of disseminated tumor cells Front Oncol 3: 320

66. Navin N, Kendall J, Troge J, Andrews P, Rodgers L, et al. (2011) Tumour evolution inferred by single-cell sequencing Nature 472: 90-94.

67. Heitzer E, Auer M, Gasch C, Pichler M, Ulz P, et al. (2013) Complex tumor genomes inferred from single circulating tumor cells by arrayCGH and next-generation sequencing. Cancer Res 73: 2965-2975.

68. Cronin M, Ross JS (2011) Comprehensive next-generation cancer genome sequencing in the era of targeted therapy and personalized oncology Biomark Med 5: 293-305.

69. Azizi E, Wicha MS (2013) Point: cancer stem cells--the evidence accumulates Clin Chem 59: 205-207.
70. Lagadec C, Vlashi E, Della Donna L, Meng Y, Dekmezian C, et al. (2010) Survival and self-renewing capacity of breast cancer initiating cells during fractionated radiation treatment. Breast Cancer Res 12: 13.

71. Phillips TM, McBride WH, Pajonk F (2006) The response of CD24(-/ low)/CD44+ breast cancer-initiating cells to radiation J Natl Cancer Inst 98: 1777-1785.

72. Karimi-Busheri F, Rasouli-Nia A, Mackey JR, Weinfeld M (2010) Senescence evasion by MCF-7 human breast tumor-initiating cells Breast Cancer Res 12: 31.

73. Diehn M, Cho RW, Lobo NA, Kalisky T, Dorie MJ, et al. (2009) Association of reactive oxygen species levels and radioresistance in cancer stem cells Nature 458: 780-783.

74. van Es JH, Clevers H (2005) Notch and Wnt inhibitors as potential new drugs for intestinal neoplastic disease Trends Mol Med 11: 496-502.

75. Stefanska B, Cheishvili D, Suderman M, Arakelian A, Huang J, et al. (2014) Genome-wide study of hypomethylated and induced genes in patients with liver cancer unravels novel anticancer targets Clin Cancer Res 20: 3118-3132.

76. Kim EH, Lee S, Park J, Lee K, Bhak J, et al. (2014) New lung cancer panel for high-throughput targeted resequencing Genomics Inform 12: 50-57.

77. Han SW, Kim HP, Shin JY, Jeong EG, Lee WC, et al. (2013) Targeted sequencing of cancer-related genes in colorectal cancer using nextgeneration sequencing PLoS One 8: e64271.

78. Zhang Y, Calado R, Rao M, Hong JA, Meeker AK, et al. (2014) Telomerase Variant A279T Induces Telomere Dysfunction and Inhibits Non-Canonical Telomerase Activity in Esophageal Carcinomas. PLoS One 9: 101010.

79. Ji Z, Su J, Liu C, Wang H, Huang D, et al. (2014) Integrating genomics and proteomics data to predict drug effects using binary linear programming PLoS One 9: 102798

80. Hallberg B, Palmer RH (2010) Crizotinib--latest champion in the cancer wars? N Engl J Med 363: 1760-1762.

81. Butrynski JE, D'Adamo DR, Hornick JL, Dal Cin P, Antonescu CR, et al. (2010) Crizotinib in ALK-rearranged inflammatory myofibroblastic tumor N Engl J Med 363: 1727-1733.

82. Smalley KS (2010) PLX-4032, a small-molecule B-Raf inhibitor for the potential treatment of malignant melanoma Curr Opin Investig Drugs 11: 699-706.

83. Campbell JP, Karolak MR, Ma Y, Perrien DS, Masood-Campbell SK, et al. (2012) Stimulation of host bone marrow stromal cells by sympathetic nerves promotes breast cancer bone metastasis in mice PLoS Biol 10: 1001363.

84. Han S, Brenner JC, Sabolch A, Jackson W, Speers C, et al. (2013) Targeted radiosensitization of ETS fusion-positive prostate cancer through PARP1 inhibition Neoplasia 15: 1207-1217.

85. Natrajan R, Wilkerson PM, Marchio C, Piscuoglio S, Ng CK, et al. (2014) Characterization of the genomic features and expressed fusion genes in micropapillary carcinomas of the breast. The Journal of pathology 232: 553-565.

86. Amorim M, Fernandes G, Oliveira P, Martins-de-Souza D, Dias-Neto E, et al. (2014) The overexpression of a single oncogene (ERBB2/HER2) alters the proteomic landscape of extracellular vesicles Proteomics 14: 1472-1479.

87. Ithimakin S, Day KC, Malik F, Zen Q, Dawsey SJ, et al. (2013) HER2 drives luminal breast cancer stem cells in the absence of HER2 amplification: implications for efficacy of adjuvant trastuzumab. Cancer Res 73: 1635-1646. 
88. Korkaya H, Wicha MS (2013) HER2 and breast cancer stem cells: more than meets the eye Cancer Res 73: 3489-3493.

89. Montemurro F, Donadio M, Clavarezza M, Redana S, Jacomuzzi ME, et al. (2006) Outcome of patients with HER2-positive advanced breast cancer progressing during trastuzumab-based therapy. The oncologist 11 : 318-324.

90. Slamon DJ, Leyland-Jones B, Shak S, Fuchs H, Paton V, et al. (2001) Use of chemotherapy plus a monoclonal antibody against HER2 for metastatic breast cancer that overexpresses HER2 N Engl J Med 344: 783-792.

91. Korkaya H, Kim GI, Davis A, Malik F, Henry NL, et al. (2012) Activation of an IL6 inflammatory loop mediates trastuzumab resistance in HER2+ breast cancer by expanding the cancer stem cell population. See comment in PubMed Commons below Mol Cell 47: 570-584

92. Singh JK, Farnie G, Bundred NJ, Simões BM, Shergill A, et al. (2013) Targeting CXCR1/2 significantly reduces breast cancer stem cell activity and increases the efficacy of inhibiting HER2 via HER2-dependent and independent mechanisms Clin Cancer Res 19: 643-656.

93. Li X, Lewis MT, Huang J, Gutierrez C, Osborne CK, et al. (2008) Intrinsic resistance of tumorigenic breast cancer cells to chemotherapy. J Natl Cancer Inst 100: 672-679.

94. Cameron D, Casey M, Press M, Lindquist D, Pienkowski T, et al. (2008) A phase III randomized comparison of lapatinib plus capecitabine versus capecitabine alone in women with advanced breast cancer that has progressed on trastuzumab: updated efficacy and biomarker analyses. Breast cancer research and treatment 112: 533-543.

95. Takebe N, Nguyen D, Yang SX (2014) Targeting notch signaling pathway in cancer: clinical development advances and challenges. Pharmacology \& therapeutics 141: 140-149.

96. Casale FP, Giurato G, Nassa G, Armond JW, Oates CJ, et al. (2014) Single-cell states in the estrogen response of breast cancer cell lines below PLoS One 9: 88485.

97. Ochsner SA, Steffen DL, Hilsenbeck SG, Chen ES, Watkins C, et al. (2009) GEMS (Gene Expression MetaSignatures), a Web resource for querying meta-analysis of expression microarray datasets: 17betaestradiol in MCF-7 cells Cancer Res 69: 23-26.

98. Zong C, Lu S, Chapman AR, Xie XS (2012) Genome-wide detection of single-nucleotide and copy-number variations of a single human cell. Science 338: 1622-1626.
99. Gundry M, Li W, Maqbool SB, Vijg J (2012) Direct, genome-wide assessment of DNA mutations in single cells Nucleic Acids Res 40: 2032-2040.

100. Voet T, Kumar P, Van Loo P, Cooke SL, Marshall J, et al. (2013) Singlecell paired-end genome sequencing reveals structural variation per cell cycle. Nucleic acids research 41: 6119-6138.

101. Chen G, Weng NP (2012) Analyzing the phenotypic and functional complexity of lymphocytes using CyTOF (cytometry by time-of-flight) Cell Mol Immunol 9: 322-323.

102. Everley RA, Kunz RC, McAllister FE, Gygi SP (2013) Increasing throughput in targeted proteomics assays: 54-plex quantitation in a single mass spectrometry run Anal Chem 85: 5340-5346.

103. Giesen C, Wang HA, Schapiro D, Zivanovic N, Jacobs A, et al. (2014) Highly multiplexed imaging of tumor tissues with subcellular resolution by mass cytometry Nat Methods 11: 417-422.

104. Zenobi R (2013) Single-cell metabolomics: analytical and biological perspectives Science 342:1243259.

105. Guo H, Zhu P, Wu X, Li X, Wen L, et al. (2013) Single-cell methylome landscapes of mouse embryonic stem cells and early embryos analyzed using reduced representation bisulfite sequencing. Genome research 23: 2126-2135.

106. Lee EJ, Luo J, Wilson JM, Shi H (2013) Analyzing the cancer methylome through targeted bisulfite sequencing Cancer Lett 340: 171-178.

107. Kaushik AK, Vareed SK, Basu S, Putluri V, Putluri N, et al. (2014) Metabolomic profiling identifies biochemical pathways associated with castration-resistant prostate cancer. Journal of proteome research 13: 1088-1100.

108. Shin SY, Fauman EB, Petersen AK, Krumsiek J, Santos R, et al. (2014) An atlas of genetic influences on human blood metabolites. Nat Genet.

109. Liu F, Zhou Y, Zhou D, Kan M, Niu X, et al. (2014) Whole DNA methylome profiling in lung cancer cells before and after epithelial-tomesenchymal transition Diagn Pathol 9: 66. 